\title{
Is Fitbit Charge 2 a feasible instrument to monitor daily physical activity and handbike training in persons with spinal cord injury? A pilot study
}

\author{
M. C. Maijers ${ }^{1} \cdot$ O. Verschuren ${ }^{1} \cdot$ J. M. Stolwijk-Swüste ${ }^{1}$ C. F. van Koppenhagen ${ }^{2} \cdot$ S. de Groot ${ }^{3,4} \cdot$ M. W. M. Post ${ }^{1,5}$
}

Received: 14 May 2018 / Revised: 16 July 2018 / Accepted: 17 July 2018

(c) International Spinal Cord Society 2018

\begin{abstract}
Study design It is a longitudinal pilot study.

Objectives To investigate the feasibility of a low-cost and widely used fitness tracker with step count and heart rate data to monitor daily physical activity in wheelchair users with spinal cord injury (SCI).

Setting Dutch community.

Methods Six participants with SCI who were in training for a handbike event were recruited. They were asked to wear a Fitbit Charge $2^{\circ} 24 \mathrm{~h}$ a day for at least 2 weeks and were questioned about the utility and user-friendliness of this device. Results Five out of six participants managed to wear the device nonstop for 2 weeks, and continued to wear the device after this initial period. Most participants were enthusiastic about the direct feedback provided by the tracker and reported the data to be accurate. Data collected during more than 2 months of three participants and during 8 months on one of them showed the possibility of detecting training days and observing interpersonal and intrapersonal variation in daily physical activity level.

Conclusions A commercially available, low-cost, self-monitoring multi-sensor wrist device or a fitness tracker like the Fitbit Charge $2^{\circ}$ can be a promising instrument to monitor daily activity levels among wheelchair users with SCI. The free commercial dashboard and log data clearly show trends of variations in physical activity and increases in heart rate, which are of value to both researchers and clinicians interested in identifying training schedules of wheelchair athletes.
\end{abstract}

M. W. M. Post

m.post@dehoogstraat.nl

1 Center of Excellence for Rehabilitation Medicine, Brain Center Rudolf Magnus, University Medical Center Utrecht, Utrecht University IDe Hoogstraat Rehabilitation, Utrecht, The Netherlands

2 Physical Medicine and Rehabilitation in University Medical Centre, Utrecht, The Netherlands

3 Amsterdam Rehabilitation Research Center I Reade, Amsterdam, The Netherlands

4 Center for Human Movement Sciences, University of Groningen, University Medical Center Groningen, Groningen, The Netherlands

5 Department of Rehabilitation Medicine, University of Groningen, University Medical Center Groningen, Groningen, The Netherlands

\section{Introduction}

The benefits of physical activity for health and well-being have been well established for able-bodied people, as well as for persons with spinal cord injury (SCI) who use a wheelchair for everyday mobility [1-3]. This last group often shows lower levels of daily physical activity and participation in sports. Therefore, the importance of stimulating physical exercise and daily activity has gained recognition [4-6].

Guiding physical training or an active lifestyle of people with SCI is often hampered by lack of accurate feedback about the actual amount of training or physical activity. A self-report of training activities has been proven unreliable in able-bodied persons and wheelchair users alike [7, 8]. It is therefore recommended to measure physical activity using movement sensors [9]. However, monitoring physical activity in manual wheelchair users, poses specific challenges for movement registration [10-12]. Multiple accelerometers, mounted to the wheelchair and the body have 
been used to measure wheelchair-driving accurately [1215]. The limitations of these devices are that the systems on the wheelchair and body are complex. Their use during daily activities and training activities in particular is unpractical. Physical activity monitors were able to distinguish between active wheelchair propulsion and other nonpropulsive wheelchair-related activities in a laboratory setting [16]. Compliance in long-term use to monitor daily activities in free-living conditions, including training activities has been a problem in previous research on accelerometers, as well as activity monitors in behavioral change programs in people with SCI [17].

In the general population, low-cost, easy-to-wear, commercially available activity trackers, including heart rate detection at the wrist have become widely used for monitoring training or daily activities to support a healthier lifestyle [18]. Recent evidence suggests that the provision of a fitness tracker is an ineffective intervention to support weight loss [19]. However, health is a more complex construct than merely body composition alone. Other researchers have concluded that these multi-sensor trackers, by combining movement and heart rate data, can detect gross increases in energy expenditure during exercise, making these wearables useful for detecting changed behavior in activity levels and possibly monitoring of training time [20]. These self-monitoring multi-sensor wearables monitor heart rate and step-count, the latter being inappropriate to our population with SCI. However, research has shown that it might be worthwhile exploring commercially available multi-sensor activity trackers, including heart rate detection also in wheelchair users [9]. Although the algorithms used to calculate step-counts and energy expenditure in these consumer-level activity trackers are based on ambulatory individuals, they have also shown to detect variations in physical activity in healthy individuals who use manual wheelchairs and arm crank ergometers at higher movement frequencies [21]. While this has not yet been tested in experienced manual wheelchair users with SCI, it seems possible to detect gross variations in physical activities with these activity trackers in wheelchair users. Therefore, we believe that they might also be a useful instrument to monitor training activities in this population, even when their physical activity does not include actual steps. As step-count trends are the main output used on online platforms of athletes, we wondered if our participants can still benefit from the motivation of comparing their step-counts and trends in physical activity levels with peers who may or may not be wheelchair users.

The HandbikeBattle (HBB) is an annual mountain timetrial for handcyclists in Austria in June. Participants are former patients of one of 12 rehabilitation centers in the Netherlands. They follow an individualized training program and are guided by health professionals from the centers during a training period of about 6 months before this event [22]. During this time, they record their training activity in an online diary. Being involved in the training of the HBB team from the region of Utrecht, we wanted to get insight into the training schedules of our participants and also into their overall daily physical activity during this training period. We aimed to determine if a commercially available activity tracker with heart rate detection could be used for this goal.

Therefore, the aim of our pilot study was to investigate the feasibility of using a commercial multi-sensor selfmonitoring wrist wearable to monitor physical activities in a group of participants with SCI from the region Utrecht while training for the HBB 2017.

\section{Methods}

\section{Design}

Longitudinal pilot study.

\section{Participants}

All six participants with SCI of the HBB 2017 team of De Hoogstraat Rehabilitation in Utrecht were invited for this study. The study protocol was approved by the Institutional Review Board of De Hoogstraat Rehabilitation and participants signed an informed consent form for the national research group that is involved in the HBB [22].

\section{Instruments}

As part of the HBB, all participants were asked to log their training activities in a digital diary, using an online log, e.g., Strava ${ }^{\circ}$ or another digital program [22]. Strava is an online social network for athletes to log training in distance, time, velocity, and altitude. One participant synchronized Strava with Fitbit after 6 months of usage. All participants underwent a medical screening before their training started, which included a Lausanne statement, physical exam, an ECG, and a graded exercise test with peak oxygen uptake (VO2peak) measurement. The protocol of this test is described in detail by Hoekstra et al. [22].

We used the Fitbit Charge $2^{\circ}$ (San Francisco, CA) as an example of a low-cost, multi-sensor, self-monitoring wrist device available on the consumer market (Fig. 1). Fitbit is the most commonly used brand regarding movement sensors in the Netherlands and the Fitbit Charge $2^{\circ}$ has proven to be a useful and reliable activity tracker in medical research previously in patients with chronic conditions other than SCI [23-28]. The arm or wrist is the preferred anatomic location of a fitness wearable in people with SCI, as 


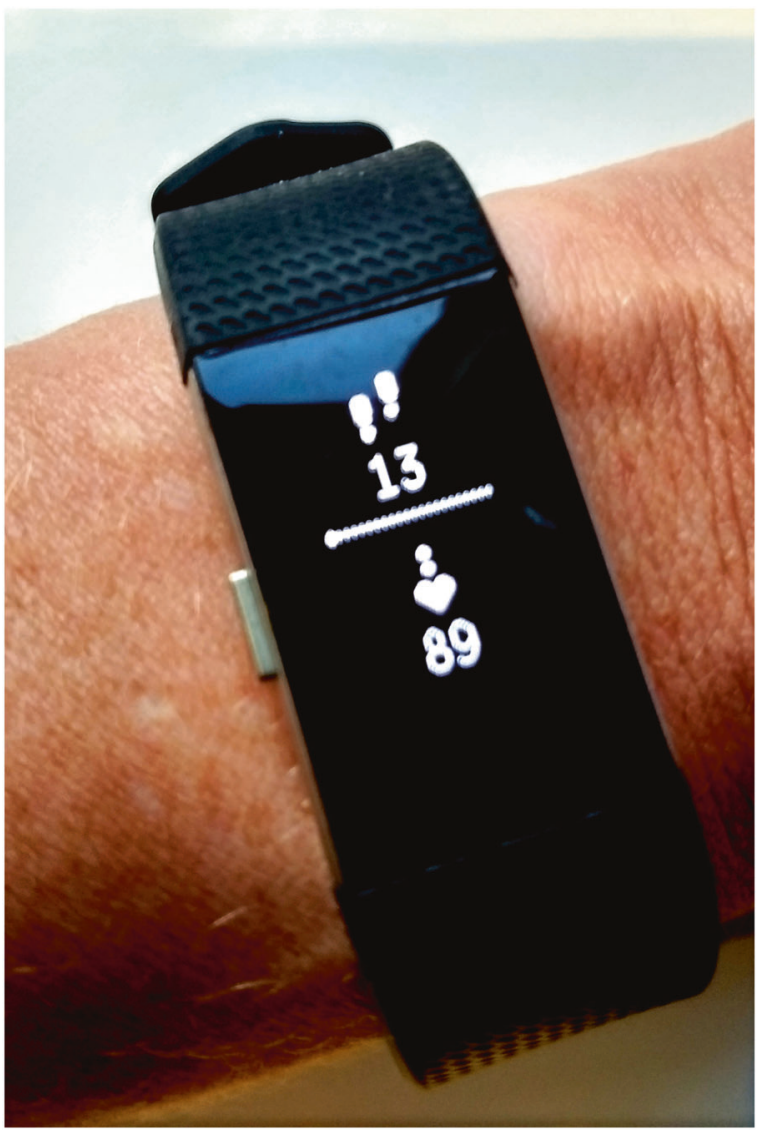

Fig. 1 Example of a Fitbit Charge $2^{\circledR}$ multi-sensor wrist device

this is the most suitable location for wheelchair users as movement often involves the upper extremities and it does not interfere with activities of daily living [13, 29, 30]. The device contains a three-axis accelerometer which tracks motion patterns, an altimeter, which tracks altitude changes, and an optical heart rate tracker, which continuously monitors heart beats. The Fitbit dashboard and log were used to obtain the Fitbit data on exercise history and progress toward a weekly exercise goal and beats per minute (BPM). The outcome data were heart rate, step-count every 5 min, and total step-count per day.

A questionnaire was designed to guide a one-to-one semi-structured interview on the usage and accuracy of the Fitbit Charge $2^{\oplus}$ by the participants. They were asked about its user-friendliness, comfort, subjective experience of the accuracy of outcome measurements, and usability of the heart rate sensor during training for the HBB.

\section{Procedure}

All our participants were provided with a Fitbit Charge $2^{\circ}$ wrist device and all participants were asked to wear it $24 \mathrm{~h}$ a day for at least 2 weeks, only taking it off when showering, swimming, and charging. The wearables were provided during a group session in which usage and benefits of training with a heart rate monitor were explained. During the group training directly after this session, all participants gained their first experience with the device. Five out of six continued to wear the Fitbit Charge $2^{\circ}$ wrist device after the 2-week period they agreed to. Three participants either bought their own Fitbit Charge $2^{\circ}$ or continued to wear the provided Fitbit Charge $2^{\circ}$ and allowed the researcher access to their data for more than 2 months, and one of them for 9 months.

A free-of-charge Fitbit account was opened for every participant that could be accessed by the participant and the researcher. The local health care professionals guiding the participants during their 6 months of training did not get access to the Fitbit data and participants were asked to continue their use of the training diaries during the course of the pilot study. The data from the training diaries, when available, were compared with the Fitbit data.

One month later at the next group training, all participants were interviewed on their experience with the usage of the wearable.

\section{Results}

The characteristics of the six participants are shown in Table 1. The results of the interviews 1 month after the distribution of the Fitbit Charge $2^{\circ}$ are summarized in Table 2. Most participants expressed their enthusiasm about the use of the Fitbit Charge $2^{\circ}$ and found the results of the total step count a valid source to objectify training activity on certain days compared to other days. One participant had limited hand function as a result of altered sensitivity unable to wear the device for more than 3 days. Figure 2 shows the total daily step-counts of these three participants during the first few months of training for the HBB. These figures are copied from the Fitbit dashboard of the participants. The figure clearly shows the intrapersonal and interpersonal differences in total step count on different days of the week. Training days are best visible in participant 2 from the end of February and participant 5 from the start in January, as on certain days their step count was approximately more than twice as high compared to other days. Participant 3 showed a different pattern, with higher step-counts on weekdays compared to weekend days, and without a clear differentiation between training days and non-training days. Figure 2 also shows interpersonal differences in total daily step-counts. Participant 3 showed by far the highest daily total step-counts, with an average of 7500 steps a day from the start of the measurements against participants 2 and 5 with a bit over 3500 and 5500 steps a day, respectively. The interviews and the training diaries of participants 2 and 5 informed us that indeed participant 2 started later with his 
Table 1 Characteristics of six HandbikeBattle participants who participated in wearing a Fitbit Charge $2{ }^{\circledR}$ wearable 24 h/day, 7 days/week, for at least 2 weeks and their baseline fitness characteristics

\begin{tabular}{|c|c|c|c|c|c|c|c|c|c|c|}
\hline No. & Age & Gender & $\begin{array}{l}\text { Time since injury } \\
\text { (months) }\end{array}$ & $\begin{array}{l}\text { Type of } \\
\text { SCI }\end{array}$ & Cause & Length (m) & Weight (kg) & $\begin{array}{l}\text { BMI }(\mathrm{kg} / \\
\left.\mathrm{m}^{2}\right)\end{array}$ & $\begin{array}{l}\mathrm{VO}_{2} \text { peak }(\mathrm{L} / \\
\mathrm{min})\end{array}$ & $\begin{array}{l}\mathrm{VO}_{2} \text { peak } / \mathrm{kg}(\mathrm{ml} / \\
\mathrm{kg} / \mathrm{min})\end{array}$ \\
\hline 1 & 65 & M & 6 & C7 AIS C & Trauma & 1.80 & 81.9 & 25.3 & 1.46 & 18 \\
\hline 2 & 55 & M & 23 & T9 AIS A & Trauma & 1.90 & 82 & 22.7 & 2.02 & 25 \\
\hline 3 & 27 & $\mathrm{~F}$ & 10 & T11 AIS A & Trauma & 1.78 & 63 & 19.9 & 1.34 & 21 \\
\hline 4 & 49 & M & 10 & T5 AIS D & Ischemic & 1.81 & 77 & 23.5 & 2.00 & 26 \\
\hline 5 & 60 & M & 58 & T3 AIS A & Trauma & 1.82 & 110 & 33.2 & 2.60 & 24 \\
\hline 6 & 52 & M & 333 & T7 AIS A & Trauma & 1.94 & 118 & 31.4 & 3.15 & 27 \\
\hline
\end{tabular}

No number of participants, $F$ female, $M$ man, $S C I$ spinal cord injury, $A I S$ ASIA Impairment Scale, $B M I$ body mass index, $V O_{2}$ peak peak oxygen consumption measured during incremental exercise test

Table 2 Results of the compliance and satisfaction questionnaire after usage of a Fitbit Charge 2 in six participants

\begin{tabular}{lllllll}
\hline Number of participants & 1 & 2 & 3 & 4 & 5 & 6 \\
\hline Still wore the Fitbit at 4 weeks? & No & Yes & Yes & Yes & Yes & Yes \\
During 24 h a day? & Yes & Yes & Yes & Yes & Yes & Yes \\
Do you believe the results to be valid? & Yes & Yes & Yes & Not always ${ }^{\mathrm{a}}$ & Yes & Yes $^{\text {b }}$ \\
Do you find the device comfortable to wear? & No & Yes & Yes & Yes & Yes & Yes \\
Did you use the HR option for training? & Yes & Yes & Yes & No, I used Garmin & Yes & Yes \\
Did you check the data online yourself? & Yes & Yes & Yes & Yes & Yes & Yes \\
\hline
\end{tabular}

$H B B$ HandbikeBattle, $d$ days, $m$ month, $w$ weeks, $H R$ heart rate

${ }^{a}$ I thought the Garmin was more reliable

${ }^{\mathrm{b}}$ Comparable to Tomtom device that I also used training schedule. Participant 5 already exercised with his handbike regularly before the start of the HBB training in January and participant 3 also used her handbike to commute to her workplace daily in addition to her training activities, which explains her lower step counts on nonworking days. She had a less active week the last week of February, when she had an infection.

Participant 3 unfortunately did not monitor her training activities in an online diary. Comparison of Fitbit data with the training diaries of participants 2 and 5 showed high agreement between both sources. Figure 3 shows an example of this high agreement between the self-reported training activities of participant 2 , as reported in the Strava ${ }^{\circ}$ app compared to the Fitbit total daily step count. He typically only trained on Saturdays and Sundays during this month.

Combining data on acceleration and heart rate gives a more detailed insight into the intensity of the physical activity or training, as illustrated in Fig. 4. Figure 4 left side shows the step-count and intensity of one day out of the month displayed on the right side. It shows quite clearly the start and ending of handbike training as both heart rate and step count increase and decrease simultaneously.

Participant 2 volunteered to wear his Fitbit Charge $2^{\circ}$ for 9 months $24 \mathrm{~h}$ a day, until several weeks after the HBB in June 2017. These data are shown in Figs. 5 and 6. Figure 5 shows the increase in total step-counts up to the marked week of the HBB and the decrease thereafter. The horizontal dotted line shows the self-chosen goal of 5000 steps a day of participant 2. Figure 6 shows in more detail the weekly physical activities and the training effect of participating in an event like the HBB and the gradual decrease in physical activities thereafter.

\section{Discussion and conclusions}

Nonstop monitoring of physical activity with the Fitbit Charge 2 for several weeks or months was feasible in five out of six wheelchair-dependent persons with SCI. Although the algorithms of this tracker are not adjusted to non-ambulatory people, we showed the data of the Fitbit ${ }^{\circ}$ dashboard and logs to be descriptive and relatively meaningful even without knowing the appropriate units for the digits or calculated counts. Data collected during more than 2 months on three participants and during 8 months on one participant showed the possibility of detecting training days and observing interpersonal and intrapersonal variations in daily physical activity.

We found excellent compliance in wearing the device $24 \mathrm{~h}$ a day, 7 days a week (24/7) for more than 2 weeks, and even several months. Most studies on physical activity among persons with SCI limited their use of an accelerometer to 7 consecutive days and often 
Fig. 2 Daily Fitbit activity data (step count) during the first 2 months of training for three participants
Fitbit daily activity data (steps) of participant 2 from January to March 2017:

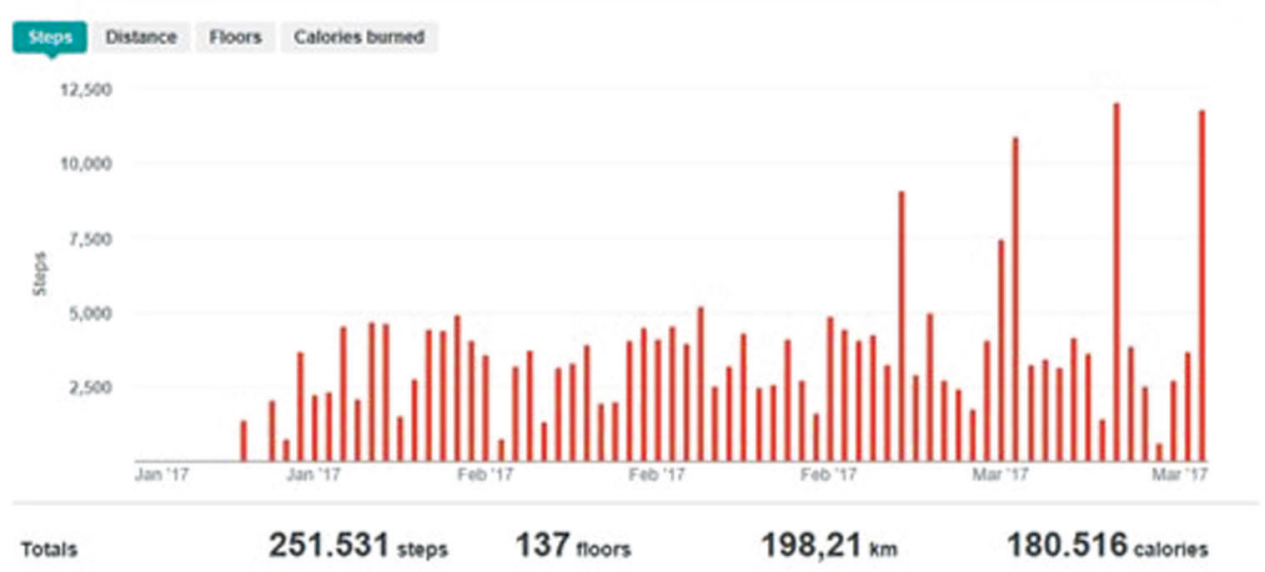

Fitbit daily activity data (steps) of participant 3 from January to March 2017:

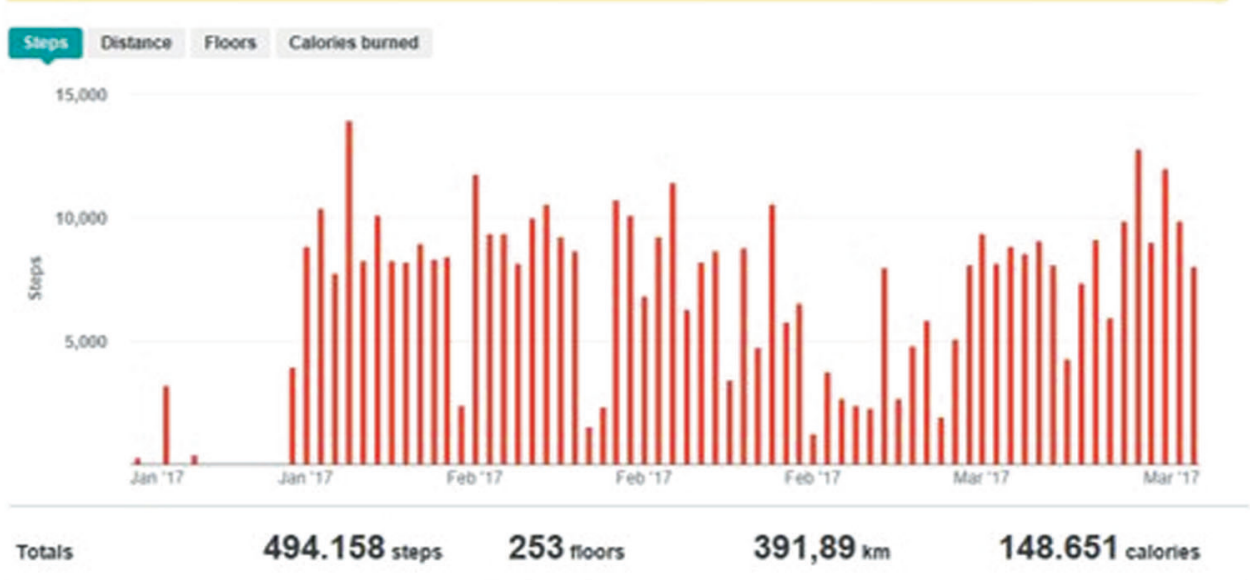

Fitbit daily activity data (steps) of participant 5 from January to March 2017:

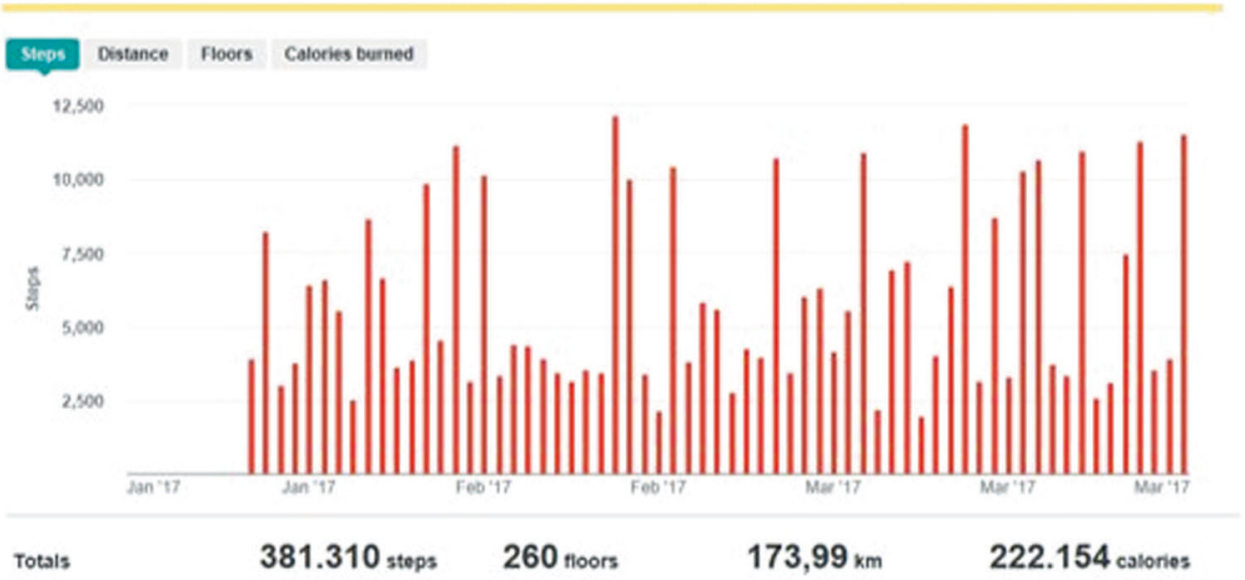

participants failed to wear the devices that are long $[7,31$, 32]. This high compliance indicates that it is possible to monitor physical activity, such as training for a sport event during long periods of time. The way of distributing the devices might contribute to the high compliance in our study. The participants were able to use the device for the first time in a training group session after an education session on the importance of training with heart rate measurement. Our participants were enthusiastic about the fact that only they had access to the data and received 
Strava activity data (self reported) of participant 2 from monday 5 March to monday 27 March 2017:

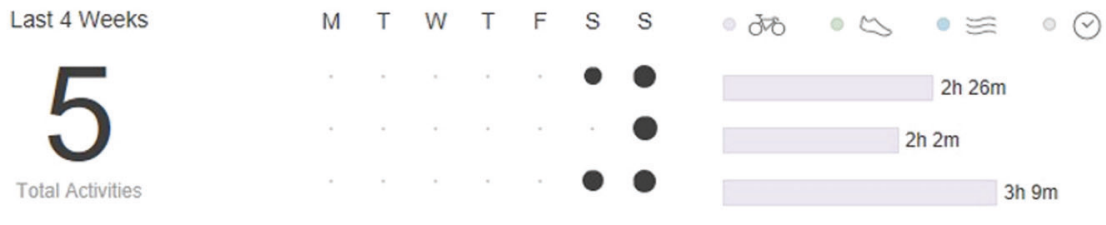

$\underline{29}$

Fitbit activity data (step count) of participant 2 from monday 5 March to monday 27 March 2017:

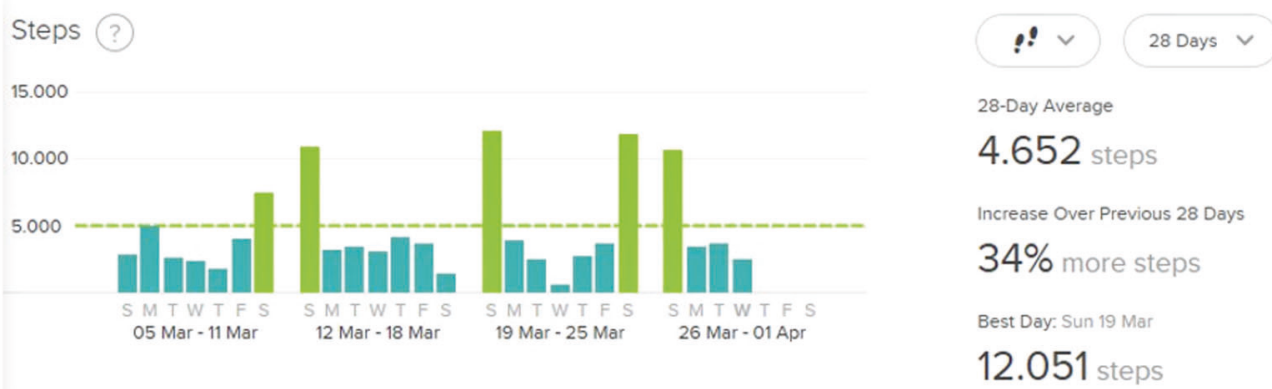

Fig. 3 Activity diary on Strava compared with activity data from Fitbit in March 2017 for participant 2

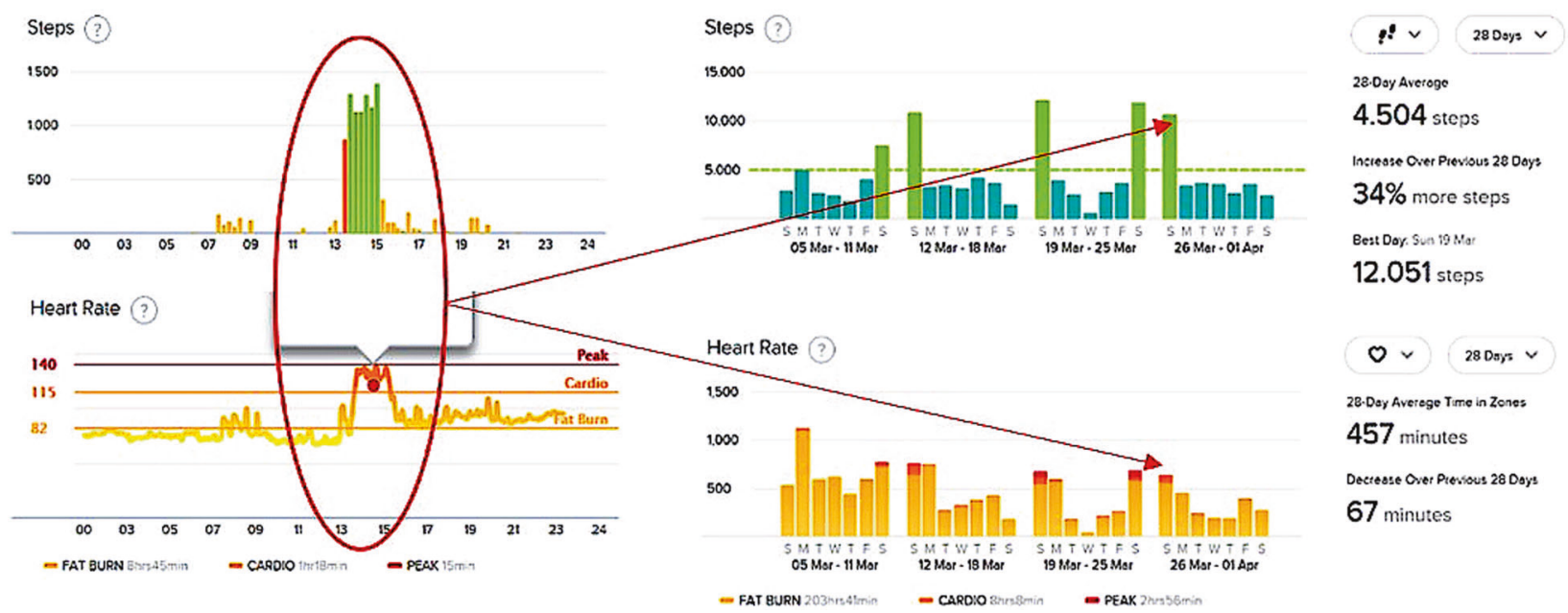

Fig. 4 Fitbit data from participant 2 of heart rate and step-count output in one day and shown in a month schedule with the days of the weeks visible by letters

immediate feedback. This is in line with self-management being an important value in the rehabilitation of persons with SCI. Previous research in wheelchair-users was mainly done with an Actigraph ${ }^{\oplus}$ accelerometer, which does not provide participants the possibility to view their own total step-count.
Although self-monitoring multi-sensor devices are widely used to monitor physical activity in the general population and in certain groups of patients [33], published experience with such devices in persons with SCI or manual wheelchair users in general is limited to a few studies in a laboratory setting with the Sense Wear Armband [34-36]. 
The advantage of using a multi-sensor device to monitor training activities as we did, is that it also measures heart rate increase. We chose the Fitbit Charge 2 as an example of a commercially available activity tracker as Fitbit wearables are widely used and there are recent reports in medical literature about its use in different patient populations [27, $37,38]$. Previous researchers found questionable results on the accuracy of the Fitbit Charge $2^{\circ}$ heart rate measurement alone [28], but combining it with movement makes it a

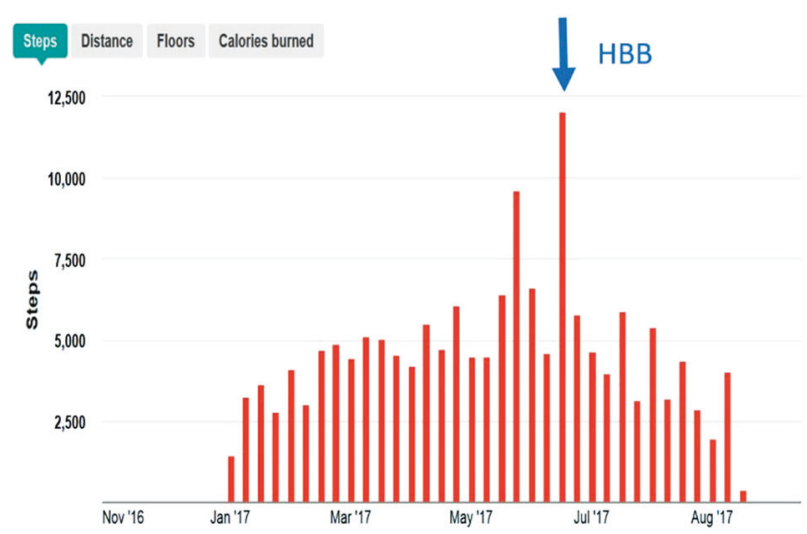

Fig. 5 Two weekly total movement count during 6 months of followup during the training for the HandbikeBattle and 2 months thereafter of participant 2. HBB HandbikeBattle useful tool when interested in long-term trends and training activities [39]. An issue when using these commercially available trackers is the privacy aspect, when logged data of patients are used in daily medical practice. Another disadvantage is that most, but not all raw data are made available for free. However, still we believe that our results show the usability when interested in trends instead of the exact energy expenditure.

In one of our participants, it proved difficult to identify training days by measurement of total step-count only. Figure 4 illustrates how combining the heart rate increase with step-count might even more accurately detect training activity in the log data. As movement detection is more challenged in wheelchair users, the benefit of heart rate increase as an indication of physical activity level and training activities might be promising in detecting trends more accurately in physical activity in this specific group.

Modern activity trackers with a heart rate sensor were developed for use in the general population. Their algorithms, which calculate the accumulation of step-counts and moderate-to-vigorous intensity physical activity are not applicable for individuals with SCI [40]. We do not measure steps in our participants in a wheelchair. Nevertheless, we have shown that with such a device, trends in physical activity can be detected in wheelchair users as well. As in the general population, these devices provide feedback to
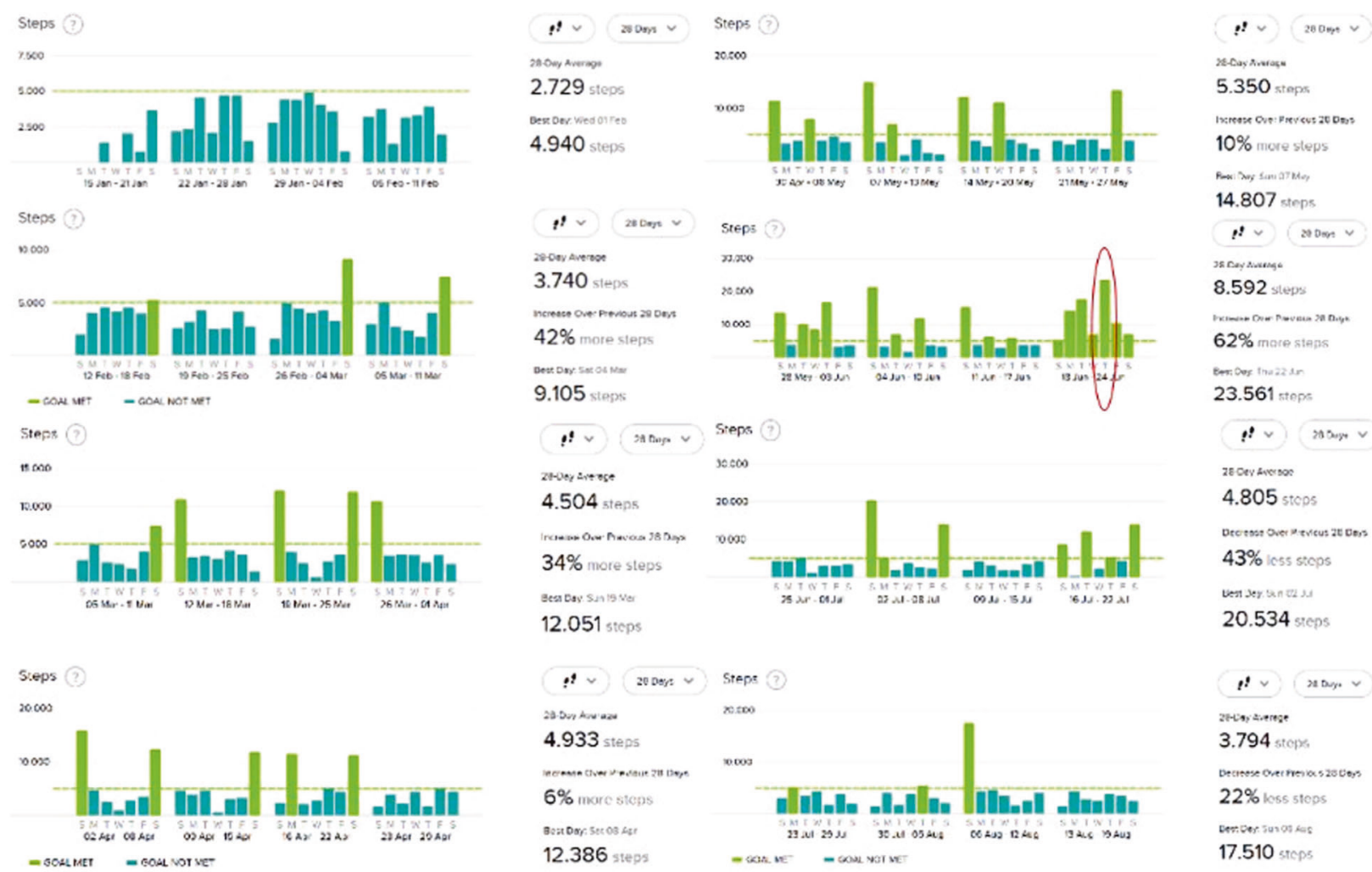

Fig. 6 Total daily step-count of participant 2 after 8 months of monitoring with the HandbikeBattle visualized by a red oval 
users in trends in energy expenditure and are most suitable for use in interventions of behavioral change [20]. Although it was not the scope of our pilot study to monitor a behavioral change in our participants, our results do illustrate how this information could be used. The data of participant 2 show that his participation in the handbike event might not have resulted in a more permanent behavioral change of regular training activity. On the other hand, the trend in physical activity could also have been the result of seasonal training differences [41]. The data of participant 3 outline the importance of combining physical exercise with daily habits or routine, like commuting to work.

The advantage of wearing a device like this $24 / 7$ is that it becomes part of a routine and will provide data 24/7, whereas self-reported diaries can be forgotten or unreliable $[42,43]$. The participants in our study were more compliant in wearing their wearable, than completing in their activity diary.

\section{Limitations and future recommendations}

Our pilot study has the limitations of a low number of participants. There is missing data on self-reported training activity, which is supported by the findings of other researchers [7]. We are aware of the fact that while industryled innovation has improved the accuracy of consumer monitors, the Fitbit Charge $2^{\circ}$, like other trackers is still not yet equivalent to the best research-grade devices or to each other [44]. We therefore acknowledge the need for quality standards. We do not know how many "steps" in a handbike differ from "steps" while wheeling a wheelchair in terms of the amount of physical activity, which would be follow-up research we would recommend pursuing. We need to study if new innovative technologies on the consumer market can be of use in our specific patient population even though they were not designed for them. We should not let the fact that new devices are released and hinder us in exploring their use and potential benefit. We further used the free available data from the manufacturer and made no effort to get access to the raw data or develop wheelchair-specific algorithms to analyze these data. Moreover, our findings are only generalizable to recreationally active athletes with SCI. We acknowledge that no conclusions are yet to be made from a single pilot study, but it does warrant investigating larger groups of wheelchair users on the use of multi-sensor wearables as a physical activity monitor.

Besides the usefulness of these devices in research settings, one can also see a role for health professionals. It was not the scope of our pilot study to intervene in the training schedule, but participant 2 could have benefited from personalized advice of a more balanced training schedule instead of training mainly on the weekends. The log data might provide a way to communicate with persons about their current physical activity and sport activity. This is of course also true for online monitoring with apps like Strava, but the activity tracker is an instrument to more accurately detect $24 / 7$ physical activity patterns. It could detect interpersonal and intrapersonal differences that explain the success of training schedules or behavioral change. We believe that output from multi-sensor devices like Fitbit Charge $2^{\circ}$ could help us evaluate treatment interventions like health education and training programs. Even in the absence of comparable data, our results show the usefulness of identifying individual patterns in daily, weekly, and even monthly quantification of physical activity. Experiences such as our own observations are important in the light of what feedback patients at risk for chronic disease or illness need in order to be motivated in healthy behavior. The fact that they can share their trends in general online platforms with friends and family improves participation in general.

In conclusion, a commercial, low-cost, easy-to-wear fitness tracker like the Fitbit Charge $2^{\circ}$ can be a promising instrument to monitor training schedules and daily activity levels among wheelchair-users with SCI. Our participants found using this device to be easy, user friendly, and the feedback to be valid and reliable.

Acknowledgements We would like to thank all participants for their involvement and feedback. We would like to thank Maremka Zwinkels for assessment of the $\mathrm{VO}_{2}$ peak.

Funding No funding was received for this work.

\section{Compliance with ethical standards}

Conflict of interest The authors declare that they have no conflict of interest.

\section{References}

1. van Koppenhagen CF, Post M, de Groot S, van Leeuwen C, van Asbeck F, Stolwijk-Swuste J, et al. Longitudinal relationship between wheelchair exercise capacity and life satisfaction in patients with spinal cord injury: a cohort study in the Netherlands. J Spinal Cord Med. 2014;37:328-37.

2. Anneken V, Hanssen-Doose A, Hirschfeld S, Scheuer T, Thietje $\mathrm{R}$. Influence of physical exercise on quality of life in individuals with spinal cord injury. Spinal Cord. 2010;48:393-9.

3. Pate RR, Pratt M, Blair SN, Haskell WL, Macera CA, Bouchard $\mathrm{C}$, et al. Physical activity and public health. A recommendation from the Centers for Disease Control and Prevention and the American College of Sports Medicine. JAMA. 1995;273:402-7.

4. WHO. Global recommendations on physical activity for health, 2010 [cited 11 Mar 2018]. http://www.who.int/dietphysicala ctivity/factsheet_recommendations/en/.

5. Haskell WL, Lee IM, Pate RR, Powell KE, Blair SN, Franklin BA, et al. Physical activity and public health: updated recommendation for adults from the American College of Sports Medicine and the American Heart Association. Circulation. 2007;116:1081-93. 
6. Martin Ginis KA, van der Scheer JW, Latimer-Cheung AE, Barrow A, Bourne C, Carruthers P. et al. Evidence-based scientific exercise guidelines for adults with spinal cord injury: an update and a new guideline. Spinal Cord. 2018;56:308-21.

7. Nooijen CF, Stam HJ, Bergen MP, Bongers-Janssen HM, Valent $\mathrm{L}$, van Langeveld $\mathrm{S}$, et al. A behavioural intervention increases physical activity in people with subacute spinal cord injury: a randomised trial. J Physiother. 2016;62:35-41.

8. Steene-Johannessen J, Anderssen SA, van der Ploeg HP, Hendriksen IJ, Donnelly AE, Brage S, et al. Are self-report measures able to define individuals as physically active or inactive? Med Sci Sports Exerc. 2016;48:235-44.

9. Nightingale TE, Rouse PC, Thompson D, Bilzon JLJ. Measurement of physical activity and energy expenditure in wheelchair users: methods, considerations and future directions. Sports Med Open. 2017;3:10.

10. Coulter EH, Dall PM, Rochester L, Hasler JP, Granat MH. Development and validation of a physical activity monitor for use on a wheelchair. Spinal Cord. 2011;49:445-50.

11. Hiremath SV, Intille SS, Kelleher A, Cooper RA, Ding D. Detection of physical activities using a physical activity monitor system for wheelchair users. Med Eng Phys. 2015;37:68-76.

12. Kooijmans H, Horemans HL, Stam HJ, Bussmann JB. Valid detection of self-propelled wheelchair driving with two accelerometers. Physiol Meas. 2014;35:2297-306.

13. Garcia-Masso X, Serra-Ano P, Garcia-Raffi LM, Sanchez-Perez EA, Lopez-Pascual J, Gonzalez LM. Validation of the use of Actigraph GT3X accelerometers to estimate energy expenditure in full time manual wheelchair users with spinal cord injury. Spinal Cord. 2013;51:898-903.

14. Kiuchi K, Inayama T, Muraoka Y, Ikemoto S, Uemura O, Mizuno K. Preliminary study for the assessment of physical activity using a triaxial accelerometer with a gyro sensor on the upper limbs of subjects with paraplegia driving a wheelchair on a treadmill. Spinal Cord. 2014;52:556-63.

15. Nightingale TE, Walhin JP, Thompson D, Bilzon JL. Influence of accelerometer type and placement on physical activity energy expenditure prediction in manual wheelchair users. PLoS One. 2015;10:e0126086

16. Leving MT, Horemans HLD, Vegter RJK, de Groot S, Bussmann JBJ, van der Woude LHV. Validity of consumer-grade activity monitor to identify manual wheelchair propulsion in standardized activities of daily living. PLoS One. 2018;13:e0194864.

17. Kooijmans H, Post MWM, Stam HJ, van der Woude LHV, Spijkerman DCM, Snoek GJ, et al. Effectiveness of a selfmanagement intervention to promote an active lifestyle in persons with long-term spinal cord injury: the HABITS Randomized Clinical Trial. Neurorehabil Neural Repair. 2017;31:991-1004.

18. Evenson KR, Goto MM, Furberg RD. Systematic review of the validity and reliability of consumer-wearable activity trackers. Int J Behav Nutr Phys Act. 2015;12:159.

19. Jakicic JM, Davis KK, Rogers RJ, King WC, Marcus MD, Helsel $\mathrm{D}$, et al. Effect of wearable technology combined with a lifestyle intervention on long-term weight loss: the IDEA Randomized Clinical Trial. JAMA. 2016;316:1161-71.

20. Price K, Bird SR, Lythgo N, Raj IS, Wong JY, Lynch C. Validation of the Fitbit One, Garmin Vivofit and Jawbone UP activity tracker in estimation of energy expenditure during treadmill walking and running. J Med Eng Technol. 2017;41:208-15.

21. Kressler J, Koeplin-Day J, Muendle B, Rosby B, Santo E, Domingo A. Accuracy and precision of consumer-level activity monitors for stroke detection during wheelchair propulsion and arm ergometry. PLoS One. 2018;13:e0191556.

22. Hoekstra S, Valent L, Gobets D, van der Woude L, de Groot S. Effects of four-month handbike training under free-living conditions on physical fitness and health in wheelchair users. Disabil Rehabil. 2017;39:1581-8.

23. Deka P, Pozehl B, Norman JF, Khazanchi D. Feasibility of using the Fitbit(R) Charge HR in validating self-reported exercise diaries in a community setting in patients with heart failure. Eur $\mathrm{J}$ Cardiovasc Nurs. 2018:1474515118766037.

24. Bai Y, Hibbing P, Mantis C, Welk GJ. Comparative evaluation of heart rate-based monitors: Apple Watch vs Fitbit Charge HR. J Sports Sci. 2018;36:1734-41.

25. Treacy D, Hassett L, Schurr K, Chagpar S, Paul SS, Sherrington C. Validity of different activity monitors to count steps in an inpatient rehabilitation setting. Phys Ther. 2017;97:581-8.

26. Lee JE, Lee DH, Oh TJ, Kim KM, Choi SH, Lim S, et al. Clinical feasibility of continuously monitored data for heart rate, physical activity, and sleeping by wearable activity trackers in patients with thyrotoxicosis: Protocol for a Prospective Longitudinal Observational Study. JMIR Res Protoc. 2018;7:e49.

27. Nogic J, Thein PM, Cameron J, Mirzaee S, Ihdayhid A, Nasis A. The utility of personal activity trackers (Fitbit Charge 2) on exercise capacity in patients post acute coronary syndrome [UPSTEP ACS Trial]: a randomised controlled trial protocol. BMC Cardiovasc Disord. 2017;17:303.

28. Benedetto S, Caldato C, Bazzan E, Greenwood DC, Pensabene V, Actis P. Assessment of the Fitbit Charge 2 for monitoring heart rate. PLoS One. 2018;13:e0192691.

29. Hiremath SV, Ding D. Evaluation of activity monitors in manual wheelchair users with paraplegia. J Spinal Cord Med. 2011;34:110-7.

30. Nightingale TE, Walhin JP, Thompson D, Bilzon JL. Predicting physical activity energy expenditure in wheelchair users with a multisensor device. BMJ Open Sport Exerc Med. 2015;1 https:// doi.org/10.1136/bmjsem-2015-000008.

31. Nightingale TE, Walhim JP, Thompson D, Bilzon JL. Predicting physical activity energy expenditure in manual wheelchair users. Med Sci Sports Exerc. 2014;46:1849-58.

32. Warms CA, Whitney JD, Belza B. Measurement and description of physical activity in adult manual wheelchair users. Disabil Health J. 2008;1:236-44.

33. Beekman E, Braun SM, Ummels D, van Vijven K, Moser A, Beurskens AJ. Validity, reliability and feasibility of commercially available activity trackers in physical therapy for people with a chronic disease: a study protocol of a mixed methods research. Pilot Feasibil Stud. 2017;3:64.

34. Conger SA, Scott SN, Fitzhugh EC, Thompson DL, Bassett DR. Validity of physical activity monitors for estimating energy expenditure during wheelchair propulsion. J Phys Act Health. 2015;12:1520-6.

35. Hiremath SV, Ding D, Farringdon J, Cooper RA. Predicting energy expenditure of manual wheelchair users with spinal cord injury using a multisensor-based activity monitor. Arch Phys Med Rehabil. 2012;93:1937-43.

36. Tanhoffer RA, Tanhoffer AI, Raymond J, Johnson NA, Hills AP, Davis GM. Energy expenditure in individuals with spinal cord injury quantified by doubly labeled water and a multi-sensor armband. J Phys Act Health. 2015;12:163-70.

37. Burton E, Hill KD, Lautenschlager NT, Thogersen-Ntoumani C, Lewin G, Boyle E, et al. Reliability and validity of two fitness tracker devices in the laboratory and home environment for older community-dwelling people. BMC Geriatr. 2018;18:103.

38. Chum J, Kim MS, Zielinski L, Bhatt M, Chung D, Yeung S, et al. Acceptability of the Fitbit in behavioural activation therapy for depression: a qualitative study. Evid Based Ment Health. 2017;20:128-33.

39. Powierza CS, Clark MD, Hughes JM, Carneiro KA, Mihalik JP. Validation of a self-monitoring tool for use in exercise therapy. PM \& R. 2017;9:1077-84. 
40. Learmonth YC, Kinnett-Hopkins D, Rice IM, Dysterheft JL, Motl RW. Accelerometer output and its association with energy expenditure during manual wheelchair propulsion. Spinal Cord. 2016;54:110-4.

41. Borisoff JF, Ripat J, Chan F. Seasonal patterns of community participation and mobility of wheelchair users over an entire year. Arch Phys Med Rehabil. 2018;99:1553-60.

42. Prince SA, Adamo KB, Hamel ME, Hardt J, Connor Gorber S, Tremblay M. A comparison of direct versus self-report measures for assessing physical activity in adults: a systematic review. Int $\mathbf{J}$ Behav Nutr Phys Act. 2008;5:56.

43. Sallis JF, Saelens BE. Assessment of physical activity by selfreport: status, limitations, and future directions. Res Q Exerc Sport. 2000;71(2 Suppl):S1-14.

44. Chowdhury EA, Western MJ, Nightingale TE, Peacock OJ, Thompson D. Assessment of laboratory and daily energy expenditure estimates from consumer multi-sensor physical activity monitors. PLoS One. 2017;12:e0171720. 